\title{
High Fundamental-Repetition-Rate Bound Solitons in Carbon Nanotube Based Fiber Lasers
}

\author{
H. H. Liu, Student Member, IEEE and K. K. Chow, Senior Member, IEEE
}

\begin{abstract}
We demonstrate bound solitons formation with high fundamental repetition rate of $211.8 \mathrm{MHz}$ from an all-fiber linear-configuration mode-locked laser incorporating a carbon-nanotube-based saturable absorber (CNT-SA) for the first time. By adjusting the polarization state of laser cavity, bound solitons exhibit a large change of pulse separation in a range of 3.5-73 times longer than the pulse width, while the phase difference of the tightly bound solitons switches between $\pm \pi / 2$. Numerical simulations confirm the experimental observations about the dependence of spectral profile of bound solitons on the pulse separation and the phase difference.
\end{abstract}

Index Terms- Optical fiber lasers, Optical solitons, Optical pulse generation.

\section{INTRODUCTION}

$\mathrm{P}$ assively mode-locked fiber lasers have emerged as one of the best pulsed light sources for a broad range of applications including frequency metrology, microscopy, and micromachining [1]. In particular, fiber lasers with high repetition rate play a key role in ultra-high speed optical communication systems. Two techniques are involved in producing high-repetition-rate pulse trains from passively mode-locked fiber lasers: one is to increase the fundamental repetition rate by shortening the laser cavity length; the other is to achieve high-order harmonic repetition rate by introducing multiple pulses with an equidistant distribution [2,3]. In order to reduce the cavity length, the thin-film-type saturable absorbers (SAs) by exploring carbon nanotubes, graphene, and semiconductor saturable absorber mirrors (SESAMs) are superior to the artificial SAs like the ones based on nonlinear polarization rotation and nonlinear amplifying loop [4,5]. So far, pulse trains with the highest fundamental repetition rate of 19.45 GHz have been produced from a 5-mm-long mode-locked fiber laser incorporating a carbon-nanotube-based saturable absorber (CNT-SA) [2]. In order to further increase the repetition rate of pulse trains, multiple pulses per cavity round trip towards harmonic mode-locking based on bound-soliton mechanism is desirable [3].

Bound solitons refer to multiple solitons that bind together as

Manuscript received xxx. This work was partially supported by Academic Research Fund Tier 2 Grant (ARC26/14) of Ministry of Education (MOE), Singapore.

The authors are with School of Electrical and Electronic Engineering, Nanyang Technological University, 50 Nanyang Avenue, Singapore 639798 (phone: +65 6790 5380; fax: +65 6793 3318; e-mail: kkchow@ntu.edu.sg). a stable unit due to a balance of repulsive and attractive forces between solitons [6]. Studies of bound solitons have attracted much research attention not only because of the benefit to understand multiple pulses operation mechanisms but also the potential for increasing telecommunication capacity in fiber transmission line [7-10]. Bound solitons have been intensively examined in most of mode-locked fiber lasers with relatively long cavity length and low fundamental repetition rate around tens of $\mathrm{MHz}$ [11-16]. In particular, it is of great interest to know if similar features of bound solitons can be obtained with high fundamental repetition rate in short-cavity mode-locked fiber lasers.

In this work, we demonstrate bound solitons formation with high fundamental repetition rate of $211.8 \mathrm{MHz}$ from a mode-locked fiber laser incorporating a CNT-SA for the first time. The CNT-SA is fabricated by the optical deposition of a CNT-film on fiber end with ring pattern, which not only enables the laser to shorten the cavity length for achieving high fundamental repetition rate, but also ensures the laser to endure high intra-cavity power for bound solitons generation. Through controlling of the intra-cavity polarization state, the pulse separation of bound solitons is found to be largely tuned by 3.5-73 times longer than the pulse width, and the phase difference of the tightly bound solitons switches between $\pm \pi / 2$. Numerical simulations are performed and confirm the experimental observations.

\section{EXPERIMENTAL SETUP}

The experimental setup of an all-fiber linear-configuration mode-locked laser incorporating a CNT-SA is shown in Fig. 1. In order to shorten the laser cavity for high repetition rate, a 976-nm laser diode and a 980/1550-nm wavelength-division multiplexing (WDM) is located outside the master oscillator. A dielectric mirror (mirror-1) is included to couple the pump into the laser cavity and extract $10 \%$ of the power at the emission wavelength from the laser cavity. A 0.16-m-long erbium-doped fiber (EDF: LIEKKI Er80) with a group velocity dispersion (GVD) parameter $\beta_{2}$ of $-0.02 \mathrm{ps}^{2} / \mathrm{m}$ is used as the gain medium. A single-mode-fiber (SMF)-based polarization controller (PC) is incorporated to match the roundtrip state of polarization. An isolator located at the output port blocks the deleterious reflection from the end-facet of the port. The total length of the fiber inside the master oscillator is $\sim 0.49 \mathrm{~m}$ and the net $\beta_{2}$ is managed to be $-0.02 \mathrm{ps}^{2}$ for soliton shaping. In the experiment, the CNTs are deposited on the end-facet of a physical contact (FC) connector by optically-driven deposition method as 
described in our previous works [17]. The deposited CNT-film has a ring pattern which enhances the thermal damage threshold of the CNT-deposited connector end. Such CNT-deposited connector end has a modulation depth of $2.6 \%$, a nonsaturable absorption of $16.3 \%$, and a saturation fluence of $47.2 \mu \mathrm{J} / \mathrm{cm}^{2}$. It is mated to a highly reflective mirror (mirror-2) to serve as a reflective CNT-SA. The spectral and temporal profiles of output pulse train are recorded by an optical spectral analyzer (Ando AQ6317B) with a resolution of $0.01 \mathrm{~nm}$ and an autocorrelator (Alnair HAC-200) with a resolution of $25 \mathrm{fs,}$ respectively.

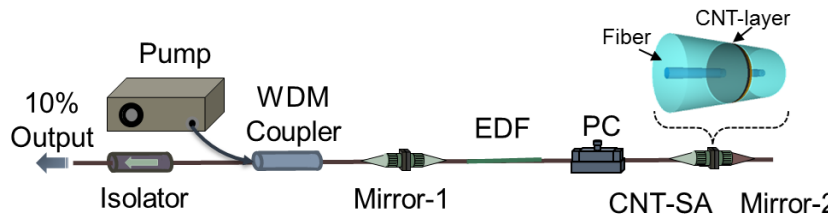

Fig. 1. Experimental setup of an all-fiber linear-configuration mode-locked laser: wavelength division multiplexing (WDM); erbium-doped fiber (EDF); polarization controller (PC); carbon-nanotube-based saturable absorber (CNT-SA).

\section{EXPERIMENTAL RESULTS}

In the experiment, the self-started mode-locking of laser occurs under a pump power of $96 \mathrm{~mW}$. Fig. 2(a) shows the corresponding output optical spectrum. The center wavelength of the spectrum locates at $1563.3 \mathrm{~nm}$ and the $3-\mathrm{dB}$ bandwidth is $\sim 4.4 \mathrm{~nm}$. As the frequency offset of Kelly-sidebands that are originated from the phase-matching resonance between soliton and dispersive wave is inversely proportional to net cavity dispersion, the developed linear-cavity mode-locked fiber laser with short cavity length and small net cavity dispersion here shows weak Kelly-sidebands superimposed on the obtained spectrum [18]. The output pulse train is depicted in the inset of Fig. 2(a). The pulse period is measured to be $\sim 4.7 \mathrm{~ns}$ which equals to one cavity round trip time. The output pulse width is measured to be around 680 fs with $\operatorname{sech}^{2}$-pulse shape fitting from the autocorrelation trace shown in Fig. 2(b). The time-bandwidth product is calculated to be around 0.368 , indicating that the output pulses are nearly transformed-limited. The RF spectrum of output pulse train is given in the inset of Fig. 2(b), showing a fundamental repetition rate of $211.8 \mathrm{MHz}$. The RF spectrum with a frequency span of $500 \mathrm{kHz}$ and a resolution of $200 \mathrm{~Hz}$ shows an extinction ratio of $83 \mathrm{~dB}$ at the

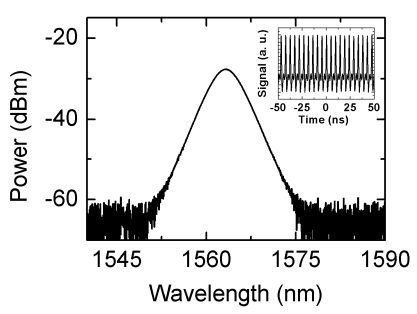

(a)

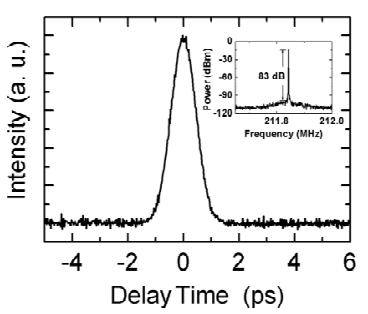

(b)
Fig. 2. Output characteristics of the mode-locked fiber laser (a) spectral profile with the inset showing optical pulse train; (b) the temporal profile with the inset showing the RF spectrum with a fundamental repetition rate of 211.8 $\mathrm{MHz}$. fundamental repetition rate, indicating that a stable mode-locking is obtained. Once the laser is mode-locked with a single pulse output per round trip, the spectral bandwidth and the pulse width are almost unchanged when the polarization state of laser is adjusted by the PC. The unchanged temporal and spectral profiles of the output pulse reveal that the intra-cavity polarization state plays a minor role in mode-locking of a laser incorporated with a CNT-SA.

Two-pulse operation per round trip is observed when the pump power exceeds $160 \mathrm{~mW}$. Such pulse splitting occurring at a high pump power can be explained by soliton-area theorem [18]. By further increasing the pump power to the maximum value of $240 \mathrm{~mW}$ with an output power of $\sim 3 \mathrm{~mW}$, bound solitons with various pulse separations are observed by adjusting the PC. Fig. 3 shows three different states of bound solitons under the pump power of $240 \mathrm{~mW}$. Different from the optical spectrum of single soliton shown in Fig. 2(a), the spectrum of bound solitons has a fringe modulation as given in Fig. 3(a). The spectral modulation period referring to the separation between two adjacent dips is estimated to be $\sim 3.3$ $\mathrm{nm}$. An asymmetrical spectral profile with a second maximum peak on the left side (i.e. blueshift) of the first maximum peak indicates that the phase difference between the bound solitons is $-\pi / 2$ [13]. In the autocorrelation trace depicted in Fig. 3(b), three peaks show an intensity ratio around $1: 2: 1$ by the cross-correlation calculation, revealing that two pulses with similar intensity and pulse width propagate close to each other. The output pulse width and the pulse separation are estimated to be $710 \mathrm{fs}$ and $2.5 \mathrm{ps}$, respectively. As the pulse separation is

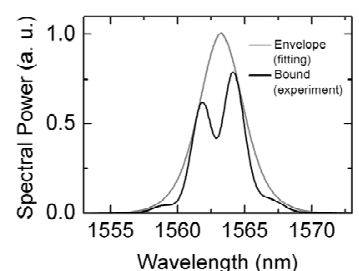

(a)

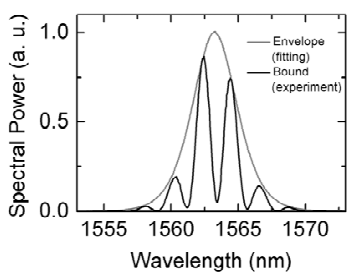

(c)

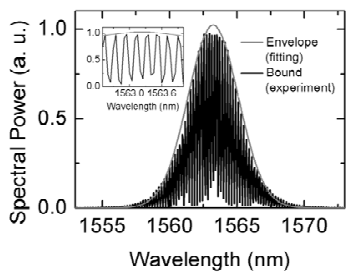

(e)

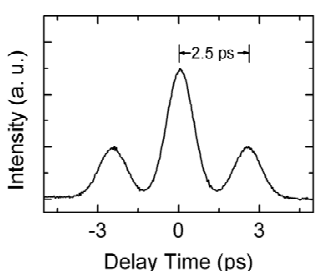

(b)

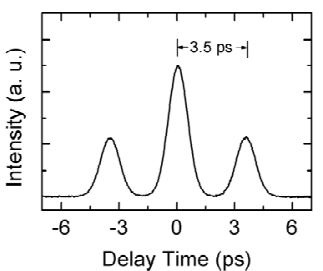

(d)

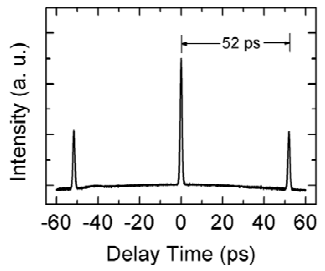

(f)
Fig. 3. Experimental results of bound solitons with various pulse separations: (a) and (b) $2.5 \mathrm{ps}$; (c) and (d) $3.5 \mathrm{ps;}$ (e) and (f) $52 \mathrm{ps}$. (a), (c), and (e) plot the normalized spectral profiles; (b), (d), and (f) show the normalized temporal profiles. The inset of (e) shows the enlarged spectral profile at peak location. 
around 3.5 times longer than the pulse width, the two pulses are bounded tightly [11]. By changing the polarization state of PC, the pulse separation is tuned correspondingly. Fig. 3(c) shows a modulation period of spectrum of $\sim 2.3 \mathrm{~nm}$ and the corresponding autocorrelation trace showing a pulse separation of $\sim 3.5$ ps is plotted in Fig. 3(d). An asymmetrical spectral profile with a second maximum peak on the right side (i.e. redshift) of the first maximum peak indicates that the phase difference between the bound solitons is around $\pi / 2$ [13]. The obtained results show that the state of bound solitons is relatively sensitive to the intra-cavity state of polarization. Although there is no polarizer adopted in the laser cavity, the inevitable existence of certain polarization dependent losses in the fiber components could lead to slight change of overall gain. Such variation of overall gain can influence the cavity nonlinearity which alters soliton interaction and results in a change of pulse separation [6].

A state of bound solitons with the largest pulse separation is obtained when the polarization state of PC is carefully adjusted. Fig. 3(e) plots the output optical spectrum with an almost symmetrical profile and a modulation period of $\sim 0.15 \mathrm{~nm}$. The corresponding autocorrelation trace showing a pulse separation of $\sim 52$ ps is given in Fig. 3(f). Since the pulse separation is extended to 73 times longer than pulse width (far greater than 5 times pulse width), the two pulses are bounded loosely. As the modulation period is very close to the spectrum resolution, it is difficult to exactly figure out the peak of the spectrum as well as the phase difference of the loosely bound solitons. Compared with the previous reports on loosely bound solitons with large pulse separation, no obvious sharp Kelly-sidebands as well as strong continuous-wave (cw) components are superimposed on the optical spectra obtained in this work [10]. By measuring the RF spectra of these states of bound solitons, almost the same signal-to-noise ratio (greater than $70 \mathrm{~dB}$ with a frequency span of $500 \mathrm{kHz}$ and a resolution of $200 \mathrm{~Hz}$ ) has been found at the fundamental repetition rate of $211.8 \mathrm{MHz}$. It is worth mentioning that the obtained bound solitons have higher fundamental repetition rate than current reports on bound solitons formation in all-fiber mode-locked lasers [11-16].

By tuning the polarization state of laser cavity, bound solitons exhibit a large change of pulse separation in a range of 2.5-52 ps which is about 3.5-73 times longer than the pulse width. Previous reports show that pulse separation of bound solitons is observed typically less than 20 ps $[8,11-13,15,19,20]$. On the contrary, the widely spaced bound solitons are experimentally obtained with their tightly bound solitons unmentioned [10,14,21]. For the first time to our knowledge, we demonstrate bound solitons generation with a widely tunable range of pulse separation from $1.55-\mu \mathrm{m}$ mode-locked fiber lasers by adjusting only polarization state. In the experiment, tightly bound solitons are easier to be observed than loosely bound solitons when the polarization state is adjusted. The tuning of pulse separation is not continuous while undergoes some lapping which is consistent with the current reports $[6,11,13,20]$. In order to figure out the dependence of spectral profile of bound solitons on the pulse separation and the phase difference as well as to understand the physical mechanism that determines the pulse separation, numerical simulation is further conducted.

\section{Simulation AND DISCUSSIONS}

Numerical simulation of a Fabry-Pérot mode-locked fiber laser is performed based on the modified nonlinear Schrödinger equation (NLSE). The cavity layout is similar to our experimental setup as shown in Fig. 1. The effects of group-velocity dispersion, Kerr effect, gain saturation, finite amplification bandwidth, and saturable absorption are considered. A piece of EDF is connected to a piece of SMF, followed by a fast SA. $10 \%$ power is exacted from the cavity by output coupler (OC). One round trip refers to passing the elements of EDF, SMF, SA, SMF, EDF, and OC in sequence. The individual elements are separately described similar to [22]. Parameters of all the elements used in the simulations are given in Table 1. In the simplified model, an initial signal is set by a sum of two weak arbitrary pulses with certain pulse separation and phase difference. Change of polarization state is represented by different initial conditions [6]. The modified NLSE is solved with a standard split-step Fourier algorithm.

TABLE I

SIMULATION PARAMETERS

\begin{tabular}{lll}
\hline \hline Symbol & \multicolumn{1}{c}{ Quantity } & \multicolumn{1}{c}{ Data } \\
\hline$L_{\text {edf }}$ & length of EDF & $0.16 \mathrm{~m}$ \\
$\beta_{2_{2} e d f}$ & GVD parameter of EDF & $-20 \mathrm{ps}^{2} / \mathrm{km}^{-1}$ \\
$\gamma_{\text {edf }}$ & nonlinear parameter of EDF & $1.06 \mathrm{~W}^{-1} \mathrm{~km}^{-1}$ \\
$g_{0}$ & small signal gain & 20 \\
$P_{\text {sat }}$ & gain saturation power & $40 \mathrm{~mW}$ \\
$\Omega_{g}$ & gain bandwidth & $10 \mathrm{THz}$ \\
$L_{s m f}$ & length of SMF & $0.33 \mathrm{~m}$ \\
$\beta_{2_{-} s m f}$ & GVD parameter of SMF & $-22 \mathrm{ps}^{2} / \mathrm{km}^{-1}$ \\
$\gamma_{\text {smf }}$ & nonlinear parameter of SMF & $2 \mathrm{~W}^{-1} \mathrm{~km}^{-1}$ \\
$a_{0}$ & modulation depth & $2.6 \%$ \\
$a_{n s}$ & non-saturable loss & $16.3 \%$ \\
$I_{\text {sat }}$ & saturation intensity & $10 \mathrm{MW} / \mathrm{cm}^{2}$ \\
\hline \hline
\end{tabular}

Fig. 4(a) shows the evolution of two pulses with an initial pulse separation of $1.5 \mathrm{ps}$ and a phase difference of $-\pi / 2$. In the evolution, two pulses are gradually built up and repel each other until their separation becomes fixed at $2.4 \mathrm{ps}$. The steady-state pulses have identical peak power with a pulse width of $\sim 520 \mathrm{fs}$. The corresponding spectrum is given in Fig. 4 (b) in which the modulation period is about $\sim 3.4 \mathrm{~nm}$. Such bound solitons with a phase difference of $-\pi / 2$ have an asymmetrical spectrum with a blueshift. Although it is difficult to estimate precisely the parameters from the real laser cavity, one can find the general agreement with the experimental observations about the dependence of spectral profile of bound solitons on the pulse separation and the phase difference. When we set the initial pulse separation between $1.5-2.4$ ps with a phase difference of $-\pi / 2$, the steady-state bound-state pulses show a minimum pulse separation of $\sim 2.4$ ps. The working mechanism of such tightly bound solitons is believed to be the direct soliton interaction at the overlapping wings [6]. The cross phase modulation-induced repulsive force between the two solitons can be balanced by the cross amplitude 


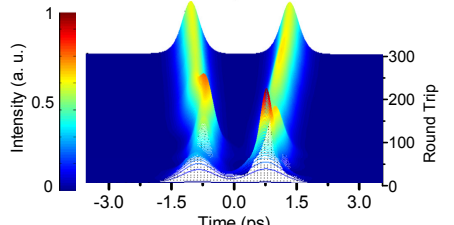

(a)

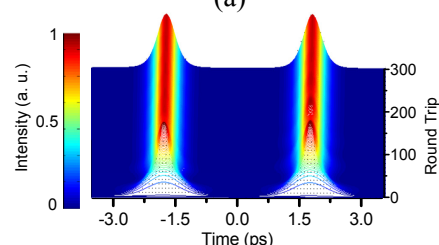

(c)

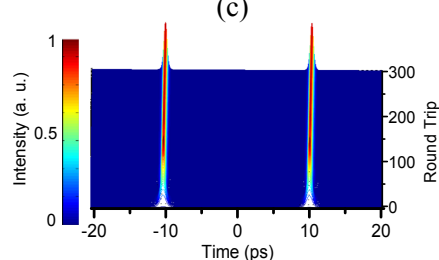

(e)

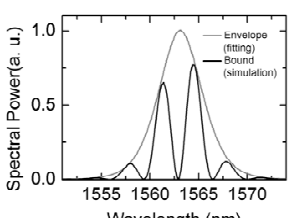

(b)

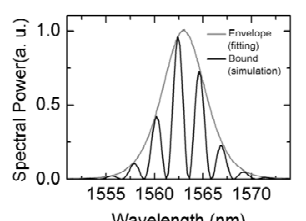

(d)

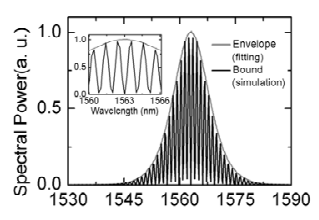

Wavelength $(\mathrm{nm})$

(f)
Fig. 4. Simulation results of bound solitons with various pulse separations: (a) and (b) 2.4 ps; (c) and (d) 3.6 ps, (e) and (f) 20 ps. (a), (c) and (e) show the evolution of two pulses; (b), (d), and(f) plot the normalized spectral profiles. The inset of (f) shows the enlarged spectral profile at the peak location.

modulation-induced attractive force, which leads to a minimum and fixed pulse separation. For initial pulse separation larger than 5 times the pulse width, the evolution of two pulses against round trip shows that the relative pulse separation remains constant as shown in Fig. 4(c). The redshift of spectrum is observed in Fig. 4(d) when the phase difference is set to be $\pi / 2$. The largely loosely bound solitons are simulated with a pulse separation of about 38 times longer than the pulse width and phase difference of $\pi$. The pulse evolution is shown in Fig. 4(e) and the corresponding spectrum is plotted in Fig. 4(f) showing a symmetrical profile with a modulation period of $\sim 0.41 \mathrm{~nm}$. The unchanged pulse separation in the evolution indicates that except sharing the cavity gain, the two solitons have very weak direct interaction. On the other hand, other effects including acoustical wave and dispersive wave always exist in fiber lasers incurred by strong electrical field of mode-locked pulses and periodical perturbations respectively. Such effects can induce long-range interaction between pulses and trap pulses in loosely bound solitons $[10,11,13]$.

\section{CONCLUSION}

We demonstrate bound solitons formation with high fundamental repetition rate of $211.8 \mathrm{MHz}$ from an all-fiber linear-configuration mode-locked laser incorporating a carbon-nanotube-based saturable absorber for the first time. By adjusting the intra-cavity polarization state, the pulse separation between the bound solitons exhibits a large change in a range of 3.5-73 times longer than the pulse width, while the phase difference of the tightly bound solitons switches between $\pm \pi / 2$. Numerical simulations confirm our experimental observations about the dependence of spectral profile of bound solitons on the pulse separation and the phase difference.

\section{REFERENCES}

[1] M. E. Fermann and I. Hartl, "Ultrafast fiber laser technology," IEEE J. Sel. Topics Quantum Electron., vol. 15, pp.191-206, Jan. 2009.

[2] A. Martinez and S. Yamashita, "Multi-gigahertz repetition rate passively mode locked fiber lasers using carbon nanotubes," Opt. Express, vol. 19, pp. 6155-6163, Mar. 2011.

[3] A. Komarov, A. Haboucha, and F. Sanchez, "Ultrahigh-repetition-rate bound-soliton harmonic passive mode-locked fiber lasers," Opt. Lett., vol. 33, pp. 2254-2256, Oct. 2008.

[4] S. Yamashita, "A tutorial on nonlinear photonic applications of carbon nanotube and graphene," J. Lightw. Technol., vol. 30, pp. 427-447, Feb. 2012.

[5] E. J. R. Kelleher, J. C. Travers, Z. Sun, A. G. Rozhin, A. C. Ferrari, S. V. Popov, and J. R. Taylor, "Nanosecond-pulse fiber lasers mode-locked with nanotubes," Appl. Phys. Lett., vol. 95, pp. 111108-1-111108-3, Sep. 2009.

[6] M. Olivier and M. Piché, "Origin of the bound states of pulses in the stretched-pulse fiber laser," Opt. Express, vol. 17, pp. 405-418, Jan. 2009.

[7] G. Martel, C. Chédot, V. Réglier, A. Hideur, B. Ortaç, and Ph. Grelu, "On the possibility of observing bound soliton pairs in a wave-breaking-free mode-locked fiber laser," Opt. Lett., vol. 32, pp. 343-345, Feb. 2007.

[8] D. Y. Tang, B. Zhao, L. M. Zhao, and H. Y. Tam, "Soliton interaction in a fiber ring laser," Phys. Rev. E, vol. 72, pp. 016616-1-016616-10, Jul. 2005.

[9] L. M. Zhao, D. Y. Tang, and D. Liu, "Ultrahigh-repetition-rate bound-soliton fiber laser," Appl. Phys. B, vol. 99, pp. 441-447, May 2010.

[10] L. Gui, X. Li, X. Xiao, H. Zhu, and C. Yang, "Widely spaced bound states in a soliton fiber laser with graphene saturable absorber," IEEE Photon. Technol. Lett., vol. 25, pp. 1184-1187, Jun. 2013.

[11] X. Wu, D. Y. Tang, X. N. Luan, and Q. Zhang, "Bound states of solitons in a fiber laser mode locked with carbon nanotube saturable absorber," Opt. Commun., vol. 284, pp. 3615-3618, July 2011.

[12] C. Mou, S. V. Sergeyev, A. G. Rozhin, and S. K. Turitsyn, "Bound state vector solitons with locked and precessing states of polarization," Opt. Express, vol. 21, pp. 26868-26875, Nov. 2013.

[13] L. Gui, X. Xiao, and C. Yang, "Observation of various bound solitons in a carbon-nanotube-based erbium fiber laser," J. Opt. Soc. Am. B, vol. 30, pp. 158-164, Jan. 2013.

[14] R. Gumenyuk and O. G. Okhotnikov, "Multiple solitons grouping in fiber lasers by dispersion management and nonlinearity control," J. Opt. Soc. Am. B, vol. 30, pp. 776-781, Apr. 2013.

[15] L. Yun and X. Liu, "Generation and propagation of bound-state pulses in a passively mode-locked figure-eight laser," IEEE Photon. J., vol. 4, pp. 512-519, Apr. 2012.

[16] J. Peng, L. Zhan, S. Luo, and Q. S. Shen, "Generation of soliton molecules in a normal-dispersion fiber laser," IEEE Photon. Technol. Lett., vol. 25, pp. 948-951, May 2013.

[17] H. H. Liu, Y. Yang, and K. K. Chow, "Enhancement of thermal damage threshold of carbon-nanotube-based saturable absorber by evanescent-field interaction on fiber end," Opt. Express, vol. 21, pp. 18975-78982, Aug. 2013.

[18] L. E. Nelson, D. J. Jones, K. Tamura, H. A. Haus, and E. P. Ippen, "Ultrashort-pulse fiber ring lasers," Appl. Phys. B, vol. 65, pp. 277-294, 1997.

[19] L. M. Zhao, D. Y. Tang, T. H. Cheng, H. Y. Tam, and C. Lu, "Bound states of dispersion-managed solitons in a fiber laser at near zero dispersion," Appl. Optics, vol. 46, pp. 4768-4773, Jul. 2007.

[20] Ph. Grelu, J. Béal, and J. M. Soto-Crespo, "Soliton pairs in a fiber laser: from anomalous to normal average dispersion regime," Opt. Express, vol. 11, pp. 2238-2243, Sep. 2003.

[21] B. Ortaç, A. Hideur, and M. Brunel, "Binding widely-separated pulses with a passively mode-locked Yb-doped double-clad fiber laser," Appl. Phys. B, vol. 79, pp. 185-192, 2004.

[22] H. H. Liu and K. K. Chow, "Enhanced stability of dispersion-managed mode-locked fiber lasers with near-zero net cavity dispersion by high-contrast saturable absorbers," Opt. Lett., vol. 39, pp. 150-153, Jan. 2014. 